\title{
Front Matter: Volume 9130
}

, "Front Matter: Volume 9130," Proc. SPIE 9130, Micro-Optics 2014, 913001 (19 May 2014); doi: 10.1117/12.2070458

SPIE. Event: SPIE Photonics Europe, 2014, Brussels, Belgium 


\title{
PROCEEDINGS OF SPIE
}

\section{Micro-Optics 2014}

\author{
Hugo Thienpont \\ Jürgen Mohr \\ Hans Zappe \\ Hirochika Nakajima \\ Editors
}

\section{4-16 April 2014 \\ Brussels, Belgium}

Sponsored by

SPIE

Cosponsored by

B-PHOT-Brussels Photonics Team (Belgium)

FWO_Fonds Wetenschappelijk Onderzoek (Belgium)

Brussels-Capital Region (Belgium)

Ville de Bruxelles (Belgium)

Cooperating Organisations

$\mathrm{CBO}-\mathrm{BCO}$ (Belgium)

European Laser Institute

Photonics 21 (Germany)

EOS-European Optical Society (Germany)

Published by

SPIE 
The papers included in this volume were part of the technical conference cited on the cover and title page. Papers were selected and subject to review by the editors and conference program committee. Some conference presentations may not be available for publication. The papers published in these proceedings reflect the work and thoughts of the authors and are published herein as submitted. The publisher is not responsible for the validity of the information or for any outcomes resulting from reliance thereon.

Please use the following format to cite material from this book:

Author(s), "Title of Paper," in Micro-Optics 2014, edited by Hugo Thienpont, Jürgen Mohr, Hans Zappe, Hirochika Nakajima, Proceedings of SPIE Vol. 9130 (SPIE, Bellingham, WA, 2014) Article CID Number.

ISSN: 0277-786X

ISBN: 9781628410785

Published by

SPIE

P.O. Box 10, Bellingham, Washington 98227-0010 USA

Telephone +1 3606763290 (Pacific Time) · Fax +1 3606471445

SPIE.org

Copyright (C) 2014, Society of Photo-Optical Instrumentation Engineers.

Copying of material in this book for internal or personal use, or for the internal or personal use of specific clients, beyond the fair use provisions granted by the U.S. Copyright Law is authorized by SPIE subject to payment of copying fees. The Transactional Reporting Service base fee for this volume is $\$ 18.00$ per article (or portion thereof), which should be paid directly to the Copyright Clearance Center (CCC), 222 Rosewood Drive, Danvers, MA 01923. Payment may also be made electronically through CCC Online at copyright.com. Other copying for republication, resale, advertising or promotion, or any form of systematic or multiple reproduction of any material in this book is prohibited except with permission in writing from the publisher. The CCC fee code is 0277-786X/14/\$18.00.

Printed in the United States of America.

Publication of record for individual papers is online in the SPIE Digital Library.

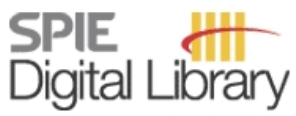

SPIEDigitalLibrary.org

Paper Numbering: Proceedings of SPIE follow an e-First publication model, with papers published first online and then in print and on CD-ROM. Papers are published as they are submitted and meet publication criteria. A unique, consistent, permanent citation identifier (CID) number is assigned to each article at the time of the first publication. Utilization of CIDs allows articles to be fully citable as soon as they are published online, and connects the same identifier to all online, print, and electronic versions of the publication. SPIE uses a six-digit CID article numbering system in which:

- The first four digits correspond to the SPIE volume number.

- The last two digits indicate publication order within the volume using a Base 36 numbering

system employing both numerals and letters. These two-number sets start with 00, 01, 02, 03, 04, $05,06,07,08,09,0 A, 0 B \ldots$. 0Z, followed by 10-1Z, 20-2Z, etc.

The CID Number appears on each page of the manuscript. The complete citation is used on the first page, and an abbreviated version on subsequent pages. Numbers in the index correspond to the last two digits of the six-digit CID Number. 


\section{Contents}

ix Conference Committee

\section{SESSION 1 DEVICES}

913002 Thermally tunable optical aperture based on a segmented thin-film resonator [9130-1] H. Block, P. Metz, J. Adam, M. Gerken, Christian-Albrechts-Univ. zu Kiel (Germany)

913003 3D optimization of a polymer MOEMS for active focusing of VCSEL beam [9130-2] S. Abada, T. Camps, B. Reig, J. B. Doucet, E. Daran, V. Bardinal, Lab. d'Analyse et d'Architecture des Systèmes, CNRS (France) and Univ. of Toulouse (France)

913004 An endoscopic microscope with liquid-tunable aspheric lenses for continuous zoom capability [9130-3]

P. Zhao, Ç. Ataman, H. Zappe, Univ. Freiburg (Germany)

913005 Design and fabrication of multiple airgap-based visible filters [9130-4]

M. Ghaderi, R. F. Wolffenbuttel, Technische Univ. Delft (Netherlands)

913006 Integrated electrochromic aperture diaphragm [9130-5]

T. Deutschmann, E. Oesterschulze, Technische Univ. Kaiserslautern (Germany)

913007 Analysis of effect of single and multiple micro-ring resonators as an optical filter using the Mason's gain formula [9130-6]

S. Kumar, A. Kumar, S. K. Raghuwanhsi, Indian School of Mines (India)

\section{SESSION 2 FABRICATION I}

913008 Wide band, wide angular width wire-grid polarizer using galvanic growth technology [9130-7]

Y. Jourlin, Lab. Hubert Curien, CNRS, Univ. de Lyon (France); M. Guttmann, Karlsruher Institut für Technologie (Germany); F. Lacour, Eurofarad (France); J. Laukkanen, Univ. of Eastern Finland (Finland); K. Yadel, F. Celle, C. Veillas, T. Kämpfe, Lab. Hubert Curien, CNRS, Univ. de Lyon (France); B. Matthis, Karlsruher Institut für Technologie (Germany); O. Parriaux, Lab. Hubert Curien, CNRS, Univ. de Lyon (France)

913009 Refractive index measurements of double-cylinder structures found in natural spider silks [9130-8]

D. J. Little, D. M. Kane, Macquarie Univ. (Australia)

9130 0A Determination of thermo-optic properties of atomic layer deposited thin $\mathrm{TiO}_{2}$ films for athermal resonant waveguide gratings by spectroscopic ellipsometry [9130-9] M. R. Saleem, Univ. of Eastern Finland (Finland) and National Univ. of Sciences and Technology (Pakistan); R. Ali, S. Honkanen, J. Turunen, Univ. of Eastern Finland (Finland) 
$9130 \mathrm{OB} \quad$ Printing on demand of polymer micro lenses array (Best Student Paper Award) [9130-10]

S. Coppola, Istituto Nazionale di Ottica, CNR (Italy) and Univ. degli Studi di Napoli "Federico II" (Italy); I. A. Grimaldi, F. Loffredo, F. Villani, G. Nenna, C. Minarini, ENEA (Italy); V. Vespini,

L. Miccio, S. Grilli, P. Ferraro, Istituto Nazionale di Ottica, CNR (Italy)

9130 OC Wavefront sensor sampling plane fabricated by maskless grayscale lithography [9130-11] G. A. Cirino, Univ. Federal de São Carlos (Brazil); F. T. Amaral, Univ. Federal de Minas Gerais (Brazil); S. A. Lopera, Univ. de São Paulo (Brazil); A. N. Montagnolil, Univ. Federal de São Carlos (Brazil); A. Arruda, Independent Consultant (Brazil); R. D. Mansano, Univ. de São Paulo (Brazil); T. M.-Brahim, Univ. de Rennes 1 (France); D. W. L. Monteiro, Univ. Federal de Minas Gerais (Brazil)

9130 OD Microfabrication and characterization of single-mask silicon microlens arrays for the IR spectra [9130-12]

P. N. A. Belmonte, D. W. de Lima Monteiro, R. F. de Oliveira Costa, Univ. Federal de Minas Gerais (Brazil); P. J. French, G. Pandraud, Technische Univ. Delft (Netherlands)

\section{SESSION 3 SENSORS AND APPLICATIONS}

9130 OE Proof-of-concept demonstration of a total internal reflection based module for fluorescence and absorbance detection using a 3D-printed syringe pump [9130-13] T. Verschooten, H. Ottevaere, M. Vervaeke, J. Van Erps, H. Thienpont, Vrije Univ. Brussel (Belgium)

9130 OG Design of a MEMS-based retina scanning system for biometric authentication [9130-15] F. Woittennek, J. Knobbe, T. Pügner, U. Schelinski, H. Grüger, Fraunhofer-Institut für Photonische Mikrosysteme (Germany)

$9130 \mathrm{OH} \quad$ Curved artificial compound-eyes for autonomous navigation [9130-16] R. Leitel, A. Brückner, W. Buß, Fraunhofer-Institut für Angewandte Optik und Feinmechanik (Germany); S. Viollet, ISM, CNRS, Aix-Marseille Univ. (France); R. Pericet-Camara, Ecole Polytechnique Fédérale de Lausanne (Switzerland); H. Mallot, Univ. of Tübingen (Germany); A. Bräuer, Fraunhofer-Institut für Angewandte Optik und Feinmechanik (Germany)

9130 0J Design, fabrication and characterization of miniaturized high resolution camera modules [9130-18]

M. Kuehn, Univ. Freiburg (Germany); M. Goetz, HSG-IMIT (Germany); C. Mueller, Univ. Freiburg (Germany); H. Reinecke, Univ. Freiburg (Germany) and HSG-IMIT (Germany)

\section{SESSION 4 FIBRES AND WAVEGUIDES}

9130 OK Self-centering fiber alignment structures for high-precision field installable single-mode fiber connectors [9130-19]

J. Van Erps, E. Ebraert, F. Gao, M. Vervaeke, F. Berghmans, Vrije Univ. Brussel (Belgium);

S. Beri, J. Watté, TE Connectivity Ltd. (Belgium); H. Thienpont, Vrije Univ. Brussel (Belgium) 
$9130 \mathrm{OL}$ Opto-mechanical design of a buckling cavity in a novel high-performance outside-plant robust field installable single-mode fibre connector [9130-21]

E. Ebraert, J. Van Erps, Vrije Univ. Brussel (Belgium); S. Beri, J. Watté, TE Connectivity Ltd. (Belgium); H. Thienpont, Vrije Univ. Brussel (Belgium)

$9130 \mathrm{OM}$ Three dimensional fabrication of optical waveguiding elements for on-chip integration [9130-22]

V. V. Parsi Sreenivas, Univ. Bremen (Germany); M. Bülters, M. Schröder, Bremer Institut für angewandte Strahltechnik GmbH (Germany); R. B. Bergmann, Univ. Bremen (Germany) and Bremer Institut für angewandte Strahltechnik GmbH (Germany)

$9130 \mathrm{ON}$ Three-dimensional buried polymer waveguides via femtosecond direct laser writing with two-photon absorption [9130-23]

D. H. H. Nguyen, K. Kaleta, S. Hengsbach, Karlsruher Institut für Technologie (Germany); U. Ostrzinski, K. Pfeiffer, micro resist technology GmbH (Germany); U. Hollenbach, J. Mohr, Karlsruher Institut für Technologie (Germany)

\section{SESSION 5 FABRICATION II}

$9130 \mathrm{OP}$ Fabrication of refractive freeform array masters for artificial compound eye cameras [9130-25]

J. Dunkel, F. Wippermann, A. Brückner, A. Reimann, M. Müller, A. Bräuer, Fraunhofer-Institut für Angewandte Optik und Feinmechanik (Germany)

9130 OQ Polymer self-assembling of light converting microlenses arrays [9130-26]

S. Coppola, Istituto Nazionale di Ottica, CNR (Italy) and Univ. degli Studi di Napoli Federico II (Italy), B. Mandracchia, Istituto Nazionale di Ottica, CNR (Italy); G. Nasti, Univ. degli Studi di Napoli Federico II (Italy) and Istituto di Chimica e Tecnologia Polimero, CNR (Italy); V. Vespini, Istituto Nazionale di Ottica, CNR (Italy); P. Pareo, CBN, Fondazione Istituto Italiano di Tecnologia (Italy); L. Carbone, NNL, Istituto Nanoscienze, CNR (Italy); M. Manca, CBN, Fondazione Istituto Italiano di Tecnologia (Italy); G. Gigli, NNL, Istituto Nanoscienze, CNR (Italy); P. Ferraro, Istituto Nazionale di Ottica, CNR (Italy)

9130 OR Uniform fabrication of thick SU-8 patterns on small-sized wafers for micro-optics applications [9130-27]

S. Abada, B. Reig, E. Daran, J. B. Doucet, T. Camps, S. Charlot, V. Bardinal, Lab. d'Analyse et d'Architecture des Systèmes, CNRS (France) and Univ. de Toulouse (France)

9130 OS Toolchain concept for the automated assembly of micro-optical systems [9130-28] S. Haag, T. Müller, Fraunhofer-Institut für Produktionstechnologie (Germany); C. Brecher, Fraunhofer-Institut für Produktionstechnologie (Germany) and RWTH Aachen (Germany)

9130 OT Design, fabrication and characterization of LVOF-based IR microspectrometers [9130-29] N. P. Ayerden, M. Ghaderi, Technische Univ. Delft (Netherlands); M. F. Silva, Univ. do Minho (Portugal); A. Emadi, Maxim Integrated (United States); P. Enoksson, Chalmers Univ. of Technology (Sweden); J. H. Correia, Univ. do Minho (Portugal); G. de Graaf, R. F. Wolffenbuttel, Technische Univ. Delft (Netherlands)

9130 OU Arrays of millimeter-sized glass lenses for miniature inspection systems [9130-30] J. Albero, S. Perrin, S. Bargiel, M. Baranski, N. Passilly, MN2S, CNRS, FEMTO-ST (France); L. Gauthier-Manuel, FEMTO-ST, CNRS (France); C. Gorecki, MN2S, CNRS, FEMTO-ST (France) 
9130 OW Linear to radial/azimuthal polarization converter in transmission using form birefringence in a segmented silicon grating manufactured by high productivity microelectronic technologies [9130-32]

T. Kaempfe, Lab. Hubert Curien, CNRS, Univ. de Lyon (France); P. Sixt, D. Renaud, A. Lagrange, F. Perrin, Lab. d'électronique et de technologie de l'information, CEA-LETI (France); O. Parriaux, Lab. Hubert Curien, CNRS, Univ. de Lyon (France)

$91300 \mathrm{X}$ Optimizing the fabrication of diffractive optical elements using a focused ion beam system [9130-35]

A. Vijayakumar, Indian Institute of Technology Madras (India); U. Eigenthaler, K. Keskinbora, Max-Planck Institut für Intelligente Systeme (Germany); G. M. Sridharan, V. Pramitha, Indian Institute of Technology Madras (India); M. Hirscher, J. P. Spatz, Max-Planck Institut für Intelligente Systeme (Germany); S. Bhattacharya, Indian Institute of Technology Madras (India)

9130 OY Fast character projection electron beam lithography for diffractive optical elements [9130-33]

T. Harzendorf, F. Fuchs, Fraunhofer-Institut für Angewandte Optik und Feinmechanik (Germany); M. Banasch, Vistec Electron Beam GmbH (Germany); U. D. Zeitner, FraunhoferInstitut für Angewandte Optik und Feinmechanik (Germany) and Friedrich-Schiller-Univ. Jena (Germany)

$9130 \mathrm{OZ}$ Chromatic confocal microscope using hybrid aspheric diffractive lenses [9130-34] M. Rayer, Heriot-Watt Univ. (United Kingdom) and AMETEK Taylor Hobson Ltd. (United Kingdom); D. Mansfield, AMETEK Taylor Hobson Ltd. (United Kingdom)

913010 Sub-wavelength grating as phase retarder: design using modal method and modeling by finite element method [9130-36]

G. M. Sridharan, S. Bhattacharya, Indian Institute of Technology Madras (India)

POSTER SESSION

913011 Approach of pullulan derivatives to resist polymers for green lithography in eco-friendly optical NEMS and MEMS [9130-37]

S. Takei, Toyama Prefectural Univ. (Japan) and Osaka Univ. (Japan); A. Oshima, Osaka Univ. (Japan); K. Ito, K. Sugahara, Toyama Prefectural Univ. (Japan); M. Kashiwakura, Osaka Univ. (Japan); T. G. Oyama, Japan Atomic Energy Agency (Japan); T. Kozawa, S. Tagawa, Osaka Univ. (Japan); M. Hanabata, Toyama Prefectural Univ. (Japan)

913012 Progressive phase conjugation and its application in reconfigurable spatial-mode extraction and conversion [9130-38]

A. Okamoto, T. Maeda, Y. Hirasaki, A. Tomita, Hokkaido Univ. (Japan); K. Sato, HokkaiGakuen Univ. (Japan)

913013 Laser-induced damage in photopolymers thin films with ultrashort pulses [9130-39] A. Žukauskas, G. Batavičiūtè, M. Ščiuka, A. Melninkaitis, M. Malinauskas, Vilnius Univ. (Lithuania) 
913014 Preliminary investigation in optical resonators based on carbon nano-tube and coupling for optoelectronics [9130-40]

P. Salzenstein, FEMTO-ST, CNRS (France); T. Makaryan, Yerevan State Univ. (Armenia)

913015 Design and analysis of beam splitters and GRIN lenses using 2D photonic crystals in silicon for telecommunications [9130-41]

U. B. M., Manipal Univ. (India); P. Nair, SSN College of Engineering (India)

913016 Tailoring the optical and rheological properties of an epoxy acrylate based host-guest system [9130-42]

U. Gleißner, Univ. Freiburg (Germany); T. Hanemann, Univ. Freiburg (Germany) and Karlsruher Institut für Technologie (Germany)

913017 Photoactivation and optogenetics with micro mirror enhanced illumination [9130-43]

F. Rückerl, Institut Pasteur, Imagopole, Plateforme d'imagerie dynamique (France);

D. Berndt, J. Heber, Fraunhofer-Institut für Photonische Mikrosysteme (Germany); S. Shorte, Institut Pasteur, Imagopole, Plateforme d'imagerie dynamique (France)

Author Index 
Proc. of SPIE Vol. $9130913001-8$

Downloaded From: https://www.spiedigitallibrary.org/conference-proceedings-of-spie on 26 Apr 2023 Terms of Use: https://www.spiedigitallibrary.org/terms-of-use 


\title{
Conference Committee
}

\author{
Symposium Chairs
}

Francis Berghmans, Vrije Universiteit Brussel (Belgium)

Ronan Burgess, European Commission (Belgium)

Jürgen Popp, Institut für Photonische Technologien e.V. (Germany)

Peter Hartmann, SCHOTT AG (Germany)

Honorary Symposium Chair

Hugo Thienpont, Vrije Universiteit Brussel (Belgium)

\section{Conference Chairs}

Hugo Thienpont, Vrije Universiteit Brussel (Belgium)

Jürgen Mohr, Karlsruher Institut für Technologie (Germany)

Hans Zappe, University of Freiburg (Germany)

Hirochika Nakajima, Waseda University (Japan)

\section{Conference Programme Committee}

Veronique Bardinal, Laboratoire d'Analyse et d'Architecture des Systèmes (France)

Francis Berghmans, Vrije Universiteit Brussel (Belgium)

Pierre H. Chavel, Laboratoire Charles Fabry (France)

Pietro Ferraro, Istituto Nazionale di Ottica (Italy)

Dietmar Fey, Friedrich-Alexander- Universität Erlangen-Nürnberg (Germany)

Michael A. Fiddy, The University of North Carolina at Charlotte (United States)

Eric Fogarassy, Institut d'Électronique du Solide et des Systèmes (France)

Alexei Glebov, OptiGrate Corporation (United States)

Jürgen Jahns, Fern Universität Hagen (Germany)

Pentti Karioja, VTT Technical Research Center of Finland (Finland)

Bernard C. Kress, Google (United States)

El-Hang Lee, Inha University (Korea, Republic of)

Marc J. Madou, University of California, Irvine (United States)

Olivier Parriaux, Laboratoire Hubert Curien (France)

Oltmann Riemer, Universität Bremen (Germany)

Clivia M. Sotomayor, Institut Català de Nanotecnologia (Spain)

Ion Stiharu, Concordia University (Canada)

Peter Van Daele, Universiteit Gent (Belgium)

Henne van Heeren, EnablingM3 (Netherlands)

Geert Van Steenberge, Universiteit Gent (Belgium) 


\section{Session Chairs}

1 Devices

Hans Zappe, University of Freiburg (Germany)

2 Fabrication I

Hans Zappe, University of Freiburg (Germany)

3 Sensors and Applications

Jürgen Mohr, Karlsruher Institut für Technologie (Germany)

$4 \quad$ Fibres and Waveguides

Jürgen Mohr, Karlsruher Institut für Technologie (Germany)

5 Fabrication II

Hugo Thienpont, Vrije Universiteit Brussel (Belgium)

6 Gratings and Diffractive Optics

Hugo Thienpont, Vrije Universiteit Brussel (Belgium) 